\title{
Heparan sulfate: lessons from knockout mice
}

\author{
Erik Forsberg and Lena Kjellén \\ Department of Medical Biochemistry and Microbiology, University of Uppsala, The Biomedical Center, Uppsala, Sweden \\ Address correspondence to: Erik Forsberg, Department of Medical Biochemistry and Microbiology, University of Uppsala, \\ The Biomedical Center, PO Box 582, SE-751 23 Uppsala, Sweden. \\ Phone: 46-18-471-4641; Fax: 46-18-471-4862; E-mail: erik.forsberg@bmc.uu.se.
}

J. Clin. Invest. 108:175-180 (2001). DOI:10.1172/JCI200113561.

Kidney agenesis, "broken heart," abnormal mast cells, somatic overgrowth, lung dysfunction, and chondrodysplasia are some phenotypes of mice where different genes important for heparan sulfate (HS) expression have been knocked out. What can we learn about HS function by studying these mice? In this Perspective we focus on data obtained so far from mouse models where the targets for the genetic modifications are the various enzymes taking part in HS biosynthesis. In addition, we will briefly discuss some mouse models in which genes for HS proteoglycan core proteins have been knocked out.

\section{HS binds to many proteins}

HS proteoglycans on cell surfaces, in basement membranes, or in cartilage (see Figure 1) influence biological processes by interacting with a large number of physiologically important macromolecules. The HS polysaccharide chains of the proteoglycans, covalently attached to different core proteins, carry negatively charged sulfate groups, whose position on the disaccharide building blocks of the polysaccharide $(\mathrm{N}$ acetylglucosamine-hexuronic acid; see Esko and Lindahl, this Perspective series, ref. 1) contributes to the specificity of the interactions. Many signaling molecules, such as FGFs, VEGF, TGF- $\beta 1$ and $-\beta 2$, as well as several chemokines and cytokines, bind to HS present on cell surface proteoglycans (2) (see Figure 1). As first demonstrated for FGFs, the proteoglycans act as coreceptors for the signaling molecules (see ref. 3 ). In addition, ECM proteins, proteases, protease inhibitors, lipases, lipoproteins, and microbial proteins are known to show affinity for HS. The pharmacologically important interaction between the protease inhibitor antithrombin and heparin (a highly sulfated variant of HS), which results in inhibition of blood coagulation, depends on several essential sulfate groups (4). In particular, the 3-O-sulfate group of the glucosamine residue in the middle of the interacting pentasaccharide sequence contributes greatly to the high-affinity binding between the polysaccharide and the protein. The interaction results in a conformational change in antithrombin, which now becomes a much more efficient inhibitor of blood coagulation. Signaling of bFGF
(FGF-2) through its receptor will not occur without binding of the growth factor to HS. In this case, 2-Osulfate groups of iduronic acid residues have been shown to be part of the interacting saccharide sequence (see Gallagher, this Perspective series, ref. 5).

\section{Gene targeting of core proteins}

and biosynthetic enzymes

Different strategies may be applied to study HS function using gene targeting. To study the role of a specific proteoglycan, the core protein of this particular proteoglycan can be knocked out. Since most cells synthesize several types of proteoglycans, HS will still be present on the cell (Figure 1). In such a model, it should be possible to investigate whether, for example, a certain syndecan core protein $(-1,-2,-3$, or -4$)$ is important for a particular signaling pathway. Gene targeting could also be used to introduce mutations in a core protein. For example, a core protein could be altered so that it no longer can function as a primer for HS biosynthesis, thus providing a system in which to study the relative contribution of the core protein and the HS chains.

Another approach is to target the genes of the various enzymes that polymerize and modify the polysaccharide chains during biosynthesis in the Golgi compartment (for details on HS biosynthesis, see Esko and Lindahl, this Perspective series, ref. 1; see also refs. 6, 7). Briefly, the biosynthesis of HS can be divided into three phases: chain initiation, polymerization, and modification. During chain initiation, linkage tetrasaccharides (glucuronic acid-galactose-galactose-xylose) are assembled on selected serine residues in the core protein. After addition of an $\mathrm{N}$-acetylglucosamine residue, polymerization takes place by the alternating addition of glucuronic acid and $\mathrm{N}$-acetylglucosamine residues. As the chain grows, modifying enzymes introduce sulfate groups at various positions, and some of the glucuronic acid residues are converted into iduronic acid. The first modifying enzyme, NDST (glucosaminyl Ndeacetylase/N-sulfotransferase), which replaces the acetyl group in $\mathrm{N}$-acetylglucosamine residues with a sulfate group, plays a key role in the biosynthesis since all other modifications only occur in the vicinity of $\mathrm{N}$ sulfate groups. The final HS biosynthesis product has 

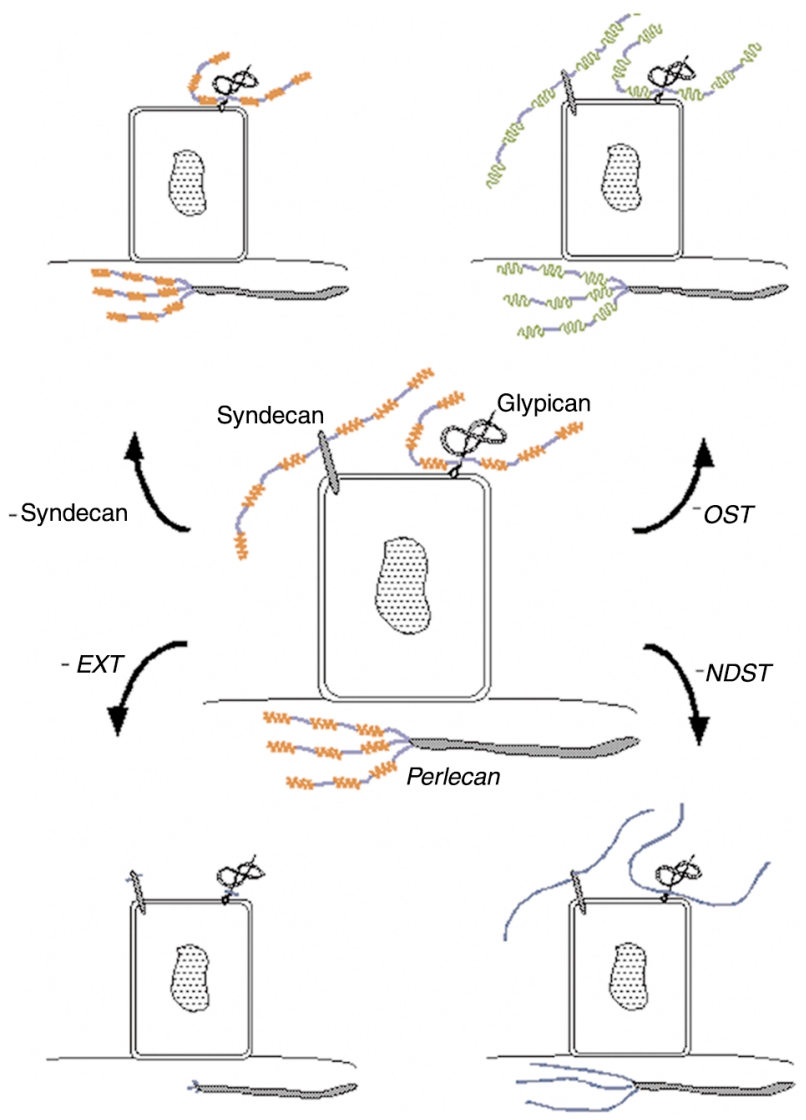

Figure 1

Targeting of genes important for HS expression. In the central part of the figure a cell and three different HS proteoglycans are shown. HS structure does not appear to be core protein-specific. Rather, HS chains of all proteoglycans synthesized by a certain cell have a similar structure. The HS chains contain sulfated regions (red) as well as unmodified regions (blue). The proteoglycan with its core protein embedded in the lipid bilayer belongs to the syndecan family (four members identified), while the other cell surface proteoglycan is a member of the glypican family (six members identified), attached to the cell surface via a GPI-anchor (1). The HS proteoglycan perlecan is found in the basement membrane underlying the cell (44). As shown in the figure, when genes of enzymes involved in polymerization of the HS chain are targeted (-EXT), only short stubs containing the HS linkage region will be attached to all three core proteins. Targeting of NDST genes (-NDST) results in the synthesis of unsulfated polysaccharide chains, whereas lack of any of the other modifying enzymes changes the fine structure of the HS chain (-OST; green) without altering its domain structure. Since several of the enzymes occur in isoforms, the alterations in HS structure may be more complex than shown in the figure. Targeting of a core protein (-Syndecan) will of course result in the lack of this particular proteoglycan at the cell surface.

a molecular design in which clusters of $\mathrm{N}$ - and $\mathrm{O}$-sulfated sugar residues (red or green in Figure 1) are separated by nonsulfated regions (blue in Figure 1).

Depending on the enzyme chosen for gene targeting, the change in HS structure may be more or less dramatic (Figure 1). If enzymes involved in polymerization of the HS chain are knocked out (EXT in Figure 1), only short stubs containing the HS linkage region will be attached to the core protein. If NDST is chosen, the polysaccharide chain will be formed but will remain nonsulfated (Figure 1). Gene targeting of any of the other modifying enzymes will result in a more subtle alteration of the structure of the HS chain (Figure 1). Since several of the enzymes involved in HS biosynthesis occur in isoforms (see Esko and Lindahl, this Perspective series, ref. 1), the outcome of a gene knockout may be more complex. For example, four NDSTs have been identified, several of which may be contained in the same cell (see ref. 8 and references therein). As demonstrated for NDST-1-deficient cells, the HS synthesized by these cells is still sulfated, but to a lesser extent than control cells (9).

So far mouse strains have been described that carry mutations in an HS polymerase (10), in two NDST isoforms $(9,11-13)$, and in a 2-O-sulfotransferase (14), respectively. In addition, work is in progress in several laboratories to create mouse strains lacking other enzymes or combinations of enzymes, as well as generating mice carrying alleles that allow for conditional gene inactivation. It can be anticipated that only the most obvious phenotypes in these mouse strains have so far been identified and that detailed analyses will reveal further physiological alterations. It will be a challenge to explain the cause of several of the observed defects on a molecular level.

\section{$\mathrm{HS}$ is necessary for gastrulation}

EXT1 and EXT2, two genes previously shown to be associated with the human hereditary multiple exostosis, are now known to possess HS polymerase activity. Both the EXT1 and EXT2 proteins harbour glucuronyl- as well as $\mathrm{N}$-acetylglucosaminyl-transferase activities (15). In vivo, the two proteins form heterodimers which are required for maximal transferase activities. EXT patients, who carry a mutation in one of the EXT genes, develop multiple benign bone tumors and often have short stature. As shown in Figure 1, a total lack of EXTs in a cell would be expected to result in truncated polysaccharide chains attached to the core proteins. Recently, a mouse strain carrying a mutation in EXT1 was described (10). Unlike EXT1 heterozygous humans, who develop exostoses in their bones at an early age, EXT1 haploinsufficient or heterozygous mice show reduced HS production and HS polymerase activity but are free of such benign tumors, even in middle age. Embryos homozygous for the mutation die at gastrulation, due to lack of an organized mesoderm and lack of extraembryonic tissues. No human patients homozygous for the EXT mutations have been identified, most likely because of similar early developmental abnormalities. ES cells from EXT1-deficient blastocysts were shown to lack HS, indicating that this is a true loss of function mutation in the EXT1 gene.

Since HS has been thought to be required for implantation, it was not expected that EXT1-deficient embryos would be able to implant into the uterine wall. Is it possible that trophectodermal cells on the surface of blastocysts still have the capacity to synthesize HS, and that cell surface HS proteoglycans on these cells are able to facilitate implantation? It has been shown that the EXT1/EXT2 heterodimer is required for efficient HS 
chain polymerization (15). However, it cannot be excluded that additional HS polymerases may exist. Alternatively, other cell surface molecules on trophectodermal cells may be able to compensate for the lack of HS.

The cause of the disturbed development in EXT1deficient embryos is not known. Most likely, the developmental defects in EXT1-deficient embryos are not primarily due to structural alterations of the basement membranes. Mouse embryos lacking the ubiquitous laminin $\gamma 1$ chain die at E5.5, due to abnormal basement membranes (16), and mice lacking the laminin/agrin/perlecan receptor dystroglycan die at E6.5 due to abnormal Reichert's membrane (17). Clearly the EXT1 phenotype differs from these two examples of severe basement membrane defects leading to early postimplantation lethality. Lin et al. (10) discuss the likely possibility that in EXT1-deficient embryos, which survive until E8.5, one or several soluble factors may be incorrectly distributed or unable to activate their receptors. In the Drosophila mutant tout-velu, which lacks a homologue to EXT1, impaired Hedgehog signaling underlies the observed developmental phenotype (ref. 18; also see Duncan et al., this Perspective series, ref. 19). Interestingly, in mice lacking EXT1, the only Hedgehog protein present at this early stage, Indian Hedgehog, is not correctly distributed in the gastrulating embryo. Whether this is a cause of the defects remains to be shown.

\section{Absence of heparin and abnormal mast cell function in mice lacking NDST-2}

After polymerization of the chain by EXT enzymes, the polysaccharide chain is modified to biologically active polymers. In 1999 Humphries et al. (12) and we (13) described the targeted inactivation of NDST-2, one of four genes whose products carry out the first step in this pathway. Since N-deacetylation and subsequent $\mathrm{N}$-sulfation of glucosamine residues is a prerequisite for all subsequent modification reactions, inactivation of this step was expected to have dramatic consequences for the structure of HS and heparin chains. However, the only obvious defect in NDST-2-null mice is that of the connective tissue-type mast cells. These cells are active during inflammatory conditions and, upon stimulation, release their granular content, including heparin, mast cell proteases, histamine, and a large number of inflammatory mediators (20). In connective tissue-type mast cells from $N D S T-2^{-/-}$mice, only a few granules can be visualized. Instead, a number of large apparently empty vacuoles are present. No sulfated heparin could be detected in these cells. In addition, mast cell proteases (chymases and tryptases) are lacking, and the amount of histamine is dramatically decreased. As a consequence of the protease deficiency, peritoneal cells from NDST-2-/ mice are no longer able to degrade fibronectin (21). Somewhat unexpectedly, neutrophils are recruited into the peritoneum of NDST-2-/ mice following mast cell activation, perhaps indicating that TNF- $\alpha$ or other cytokines are still present in the mast cells and can be secreted upon degranulation. Since NDST-2-null mice show no obvious signs of thrombo- sis, we can conclude that endogenous heparin is not involved in the regulation of blood clotting but instead has a crucial role in mast cell homeostasis.

The mast cell phenotype was expected, since NDST-2 is expressed at significant levels in these cells but NDST1 is not. NDST-2 is, however, expressed in large parts of the body both during development and in adult mice, at least at the mRNA level. Therefore, the absence of structural alterations of HS in liver as well as in kidney was surprising. Either other NDSTs can compensate for NDST-2, or their functions are redundant in many tissues and organs. Indeed, since it is not yet clear whether the NDST-2 protein is expressed in the liver and kidney, it remains possible that the other NDST isoforms are more important for HS biosynthesis in these organs.

NDST-1 is a major player in HS biosynthesis In contrast to NDST-2-deficient mice, mouse embryos carrying targeted mutations in the related enzyme NDST-1 show dramatically reduced N-sulfation of HS in most parts of the body (9). However, the only obvious phenotype so far reported is abnormal lungs ( 9 , 11); the type II epithelial cells in HS-deficient mice are increased in number but do not secrete sufficient amounts of surfactants. As a consequence, the newborn pups die shortly after birth due to lung failure in a condition resembling the respiratory distress syndrome in humans. Skull and skeletal abnormalities and defects in eye development are nonpenetrant phenotypes. Some embryos die during the prenatal period, but the defects causing the embryonic lethality have not been identified. Detailed structural analysis of embryonic liver HS from NDST-1/-- mice in our laboratory demonstrates that in addition to the lowered Nsulfation, the O-sulfation reactions and epimerization of glucuronic acid to iduronic acid occur at reduced levels (J. Ledin et al., unpublished results). This result is in line with the important role of NDSTs in HS biosynthesis, regulating further enzymatic modifications. In light of the many described functions for HS, it is intriguing that NDST-1-deficient embryos with large structural changes of HS in most basement membranes of the body still are able to complete the prenatal period. It may be speculated that the low-sulfated HS of the embryos still contain binding sites for certain key ligands, e.g., growth factors, important for embryonic development. Our observation that embryos lacking both NDST-1 and NDST-2 die during early embryogenesis (K. Holmborn et al., unpublished results) indicates that NDST-2, which is crucial for heparin formation, also plays a role in HS biosynthesis.

\section{Renal agenesis and skeletal defects}

in mice lacking 2-O-sulfotransferase

After $\mathrm{N}$-deacetylation and $\mathrm{N}$-sulfation, carried out by the NDSTs, glucuronic acid residues are epimerized to iduronic acid. A subset of these iduronic acid residues (as well as a small number of glucuronic acid residues) become 2-O-sulfated. Only one gene encoding a 2-O-sulfotransferase has been identified in the mammalian genome. A random gene trap insertion into the mouse 
2-OST gene was described in 1998 (14). Mice homozygous for the mutation show renal agenesis and die in the neonatal period. Expression of the 2-O-sulfotransferase is apparently important for mesenchymal condensation around the ureteric bud and initiation of branching morphogenesis, and the mice exhibit additional defects of the eye and skeleton. Cleft palate and polydactyly also occur in some of the homozygous mice. No human genetic disorders with defects reminiscent of the murine phenotype have so far been associated with human chromosome 1p31, where the human 2-O-sulfotransferase gene maps. If it is correct that only one 2-O-sulfotransferase gene is expressed, mice with a null mutation should completely lack 2-O-sulfated HS, as appears to be the case in analysis of HS synthesized by embryonic fibroblasts from these mutant mice (C. Merry and J. Gallagher, personal communication). The structurally altered mutant HS will most likely have different ligand binding properties than does normal HS. As with the NDST1-deficient embryos, it is notable that embryos lacking 2-O-sulfotransferase can still form most organs and tissues. Moreover, the phenotypes of these mutant strains are distinct, with cartilage and kidney defects seen in one case, and lung defects in the other.

The four mutants discussed above showed structural alterations in the polysaccharide chains of $\mathrm{HS} /$ heparin proteoglycans. It will be useful to compare these mutants with others bearing null mutations in one or another core protein. For instance, a knockout of the serglycin core protein, the only heparin-bearing core protein in connective tissue-type mast cells, may well phenocopy NDST-2-deficient mice. However, no serglycin-deficient mouse strain has yet been described.

\section{Perlecan and Schwartz-Jampel syndrome}

Embryos carrying a targeted null mutation in Perlecan show a different phenotype from that of mice lacking any described biosynthetic enzyme, as demonstrated independently by two groups $(22,23)$. Many perlecandeficient embryos die at E10-E12, due to rupture of basement membranes in the heart. Embryos that survive this critical stage go on to develop exencephaly or other brain defects and die perinatally. These defects are due to stress-sensitive basement membranes, which are obviously not correctly assembled in the absence of perlecan.

Chondrodysplasia is also observed in Perlecan mutant embryos surviving the early critical period. The phenotype is characterized by an abnormal fibrillar collagen network and altered expression of other cartilage ECM genes, consistent with the suggestion of Costell et al. that perlecan acts to protect the cartilage ECM from degradation (22). In the other report on embryos lacking perlecan, the results indicate that defective growth factor signalling may also contribute to chondrodysplasia in perlecan-null embryos (23). The structural importance of perlecan can be attributed both to the core protein, which binds to, e.g., collagen type IV and nidogen $\mathrm{I}$, and to the HS chains which interact with laminins among other ECM proteins (24). Growth factor binding primarily occurs to the HS chains, but the perlecan core protein has been shown to interact with
FGF-7 (25) and also with FGF-BP (26), a modulator of FGF-2 activity. Interestingly, Nicole et al. (27) recently identified mutations in Perlecan associated with the human Schwartz-Jampel syndrome (chondrodystrophic myotonia). However, while these patients have a myotonic phenotype and skeletal dysplasia, they do not show craniofacial abnormalities. Furthermore, the lethal deterioration of the heart has not been observed. Since the N-terminal part of perlecan is still present in patients with Schwartz-Jampel syndrome, and this region carries the HS chains, a lack of HS might be a factor influencing the craniofacial phenotypes.

Another HS proteoglycan present in most basement membranes, and for which a targeted mouse model exists, is agrin (28). The phenotype of mutants lacking the splice form of agrin, which is present in neuromuscular junctions, is restricted to these sites (29). Mutations in another basement membrane HS proteoglycan, collagen type XVIII (28), have not yet been reported, but this phenotype will be of great interest, considering that this protein contains an endostatin domain which is implicated in angiogenesis and tumor growth (see Iozzo and San Antonio, this Perspective series, ref. 30).

\section{Mutations in the cell surface proteoglycans}

The syndecan-1, syndecan-4, glypican-2, glypican-3, and glypican-4 core protein genes have now been knocked out. Syndecan-1-deficient mice are viable and fertile and develop normally (2), although healing of skin and corneal wounds are defective in these mice. When crossed with transgenic mice expressing Wnt-1 in mammary gland, hyperplasia in this tissue is greatly reduced, indicating a role for syndecan-1 in Wnt-1induced tumorigenesis (31). The role of HS proteoglycans in Wnt signaling pathways has previously been demonstrated in Drosophila, where the Wnt-1 homologue Wingless appears to be dependent on HS proteoglycans for signaling (32). This morphogen is required for patterning of the embryonic parasegments. However, in Drosophila signaling appears to occur via dally, one of two glypican homologues.

While no abnormalities have been found in glypican2 -deficient mice (33), the phenotype of the glypican3 -deficient mice is severe (34). The mice exhibit developmental overgrowth, perinatal death, cystic and dysplastic kidneys, and abnormal lung development. These features are also observed in patients with the Simpson-Golabi-Behmel syndrome, an X-linked disorder associated with mutations in the Glypican-3 gene (35). This syndrome and the Glypican-3 mouse model will be described in a separate article of this Perspective series (Filmus and Selleck, ref. 36).

Syndecan-4-deficient mice $(37,38)$ are both healthy and fertile. Syndecan-4 has previously been implicated in focal adhesion formation (39), but fibroblasts from the mutant embryos form focal adhesions normally when cultured on fibronectin. However, focal adhesion formation is impaired if the heparin-binding domain of fibronectin is added in soluble form to cells attached to the cell-binding fragment. The authors speculate that, during inflammation or wounding when fibronectin is 
degraded, syndecan-4 may be important for focal adhesion formation and actin fiber organization, which in turn contribute to cell migration. Interestingly, Echtermeyer et al. found recently that mice homozygous (or even heterozygous) for the syndecan- 4 mutation display delayed wound healing and impaired angiogenesis in the granulation tissue (38). A recent report also indicates that the control of coagulation in fetal vessels of the placenta of syndecan-4-/- mice is impaired (40).

\section{What have we learned?}

What conclusions can be drawn from results so far obtained studying the knockout mice described in this Perspective? Previous studies in Drosophila have shown that HS in this model organism is crucial for embryonic development (41). This appears to be the case in mice, too, as demonstrated, for example, by the early embryonic lethality of EXT-1-deficient mice and of embryos lacking both NDST-1 and NDST-2. The lack of kidneys in the 2-O-sulfotransferase mutant mice, the delayed maturation of lungs in $\mathrm{NDST1}^{-/-}$, and the abnormal development of lungs and kidneys in Glypican-3-1- mice also support this notion.

How do the HS proteoglycans perform their functions on a molecular level? The structural importance of perlecan, previously suggested, has now been clearly demonstrated in the perlecan knockout mice. Studies originally performed on FGF-2 have demonstrated that HS proteoglycans act as coreceptors (see Gallagher, this Perspective series, ref. 5). For FGF-2, participation of the HS proteoglycan is required not only for binding, but also for signaling. The studies of the syndecan1 -deficient mice nicely demonstrate the role of this proteoglycan in Wnt-1 signaling. The 2-O-sulfotransferase knockout mice are also interesting in this respect, since binding of FGF-2 to HS has been shown to depend on the presence of 2-O-sulfate groups (Gallagher, this series, ref. 5). However, the phenotype of the FGF-2-null mice, which are viable and apparently normal in the development of their kidneys but not their cerebral cortex, differs from that of the 2-O-sulfotransferase mutants (42). Evidently, a number of growth factors and morphogens are important for kidney development, some of which may be influenced by the altered HS structure in the 2-O-sulfotransferase-deficient embryos.

The pentasaccharide sequence interacting with antithrombin has been studied in great detail, and, as mentioned above, the 3-O-sulfate group has been shown to be essential for the interaction. However, mice that lack 3-O-sulfotransferase-1, the enzyme believed to be responsible for the 3-O-sulfation of this sequence, do not show a procoagulant phenotype (43). A more detailed characterization of these mice at the molecular level may help us to understand this unexpected finding. Extended studies of the syndecan-4-deficient mice, which appeared to display some coagulation abnormalities, should also be done. The absence of heparin in the NDST-2-deficient mice without any obvious influence on the coagulation status of the mice demonstrates that endogenous heparin does not take part in regulation of coagulation. Instead, heparin plays a role in regulating the mast cell mediators such as histamine and the mast cell-specific proteases.

The restricted phenotype of the NDST-2-deficient mice also puts it on the list of unexpected results. NDST- 1 and NDST- 2 are both widely expressed in mice. Still, gene targeting of NDST-1 has serious effects on HS structure in large parts of the body, while the absence of NDST-2 has no apparent effect on HS structure. The mouse models are clearly good reminders of the extreme complexity of the system we are trying to understand and the great gulf between in vitro studies and in vivo phenotypes.

Characterization of the different mouse strains with altered HS expression has just started. Obviously, a lot of data will be collected both from these strains and from new strains that are only now being generated. The results obtained to date have already raised the profile of HS research in fields such as skeletal and eye development. Cell lines derived from the different mutant mice will provide complementary tools to study HS biosynthesis and regulation at a molecular level. There is a great deal to learn.

\section{Acknowledgments}

We acknowledge funding from the Medical Research Council, the Swedish Natural Science Research Council, Polysackaridforskning AB, European Commission Contract QLK3-CT-1999-00536, the programme "Glycoconjugates in Biological Systems" sponsored by the Swedish Foundation for Strategic Research, Gustaf V:s 80-årsfond, Magnus Bergvalls stiftelse and Åke Wibergs stiftelse.

1. Esko, J.D., and Lindahl, U. 2001. Molecular diversity of heparan sulfate. J. Clin. Invest. 108:169-173.

2. Bernfield, M., et al. 1999. Functions of cell surface heparan sulfate proteoglycans. Annu. Rev. Biochem. 68:729-777.

3. Park, P.W., Reizes, O., and Bernfield, M. 2000. Cell surface heparan sulfate proteoglycans: selective regulators of ligand-receptor encounters. $J$. Biol. Chem. 275:29923-29926.

4. Rosenberg, R.D., Shworak, N.W., Liu, J., Schwartz, J.J., and Zhang, L. 1997. Heparan sulfate proteoglycans of the cardiovascular system. Specific structures emerge but how is synthesis regulated? J. Clin. Invest. 100:S67-S75.

5. Gallagher, J.T. 2001. Heparan sulfate: growth control with a restricted sequence menu. J. Clin. Invest. In press.

6. Sugahara, K., and Kitagawa, H. 2000. Recent advances in the study of the biosynthesis and functions of sulfated glycosaminoglycans. Curr. Opin. Struct. Biol. 10:518-527.

7. Lindahl, U., Kusche-Gullberg, M., and Kjellen, L. 1998. Regulated diversity of heparan sulfate. J. Biol. Chem. 273:24979-24982.

8. Aikawa, J., Grobe, K., Tsujimoto, M., and Esko, J.D. 2000. Multiple isozymes of heparan sulfate/heparin GlcNAc N-deacetylase/N-sulfotransferase: structure and activity of the fourth member, NDST4. J. Biol. Chem. In press.

9. Ringvall, M., et al. 2000. Defective heparan sulfate biosynthesis and neonatal lethality in mice lacking $\mathrm{N}$-deacetylase/N-sulfotransferase-1.J. Biol. Chem. 275:25926-25930.

10. Lin, X., et al. 2000. Disruption of gastrulation and heparan sulfate biosynthesis in EXT1-deficient mice. Dev. Biol. 224:299-311.

11. Fan, G., et al. 2000. Targeted disruption of NDST-1 gene leads to pulmonary hypoplasia and neonatal respiratory distress in mice. FEBS Lett. 467:7-11.

12. Humphries, D.E., et al. 1999. Heparin is essential for the storage of specific granule proteases in mast cells. Nature. 400:769-772.

13. Forsberg, E., et al. 1999. Abnormal mast cells in mice deficient in a heparin-synthesizing enzyme. Nature. 400:773-776.

14. Bullock, S.L., Fletcher, J.M., Beddington, R.S., and Wilson, V.A. 1998. Renal agenesis in mice homozygous for a gene trap mutation in the gene 
encoding heparan sulfate 2-sulfotransferase. Genes Dev. 12:1894-1906.

15. McCormick, C., Duncan, G., Goutsos, K.T., and Tufaro, F. 2000. The putative tumor suppressors EXT1 and EXT2 form a stable complex that accumulates in the Golgi apparatus and catalyzes the synthesis of heparan sulfate. Proc. Natl. Acad. Sci. USA. 97:668-673.

16. Smyth, N., et al. 1999. Absence of basement membranes after targeting the LAMC1 gene results in embryonic lethality due to failure of endoderm differentiation. J. Cell Biol. 144:151-160.

17. Williamson, R.A., et al. 1997. Dystroglycan is essential for early embryonic development: disruption of Reichert's membrane in Dag1-null mice. Hum. Mol. Genet. 6:831-841.

18. Bellaiche, Y., The, I., and Perrimon, N. 1998. Tout-velu is a Drosophila homologue of the putative tumour suppressor EXT-1 and is needed for Hh diffusion. Nature. 394:85-88.

19. Duncan, G., McCormick, C., and Tufaro, F. 2001. The link between heparan sulfate and hereditary bone disease: finding a function for the EXT family of putative tumor suppressor proteins. J. Clin. Invest. In press. 20. Galli, S.J. 2000. Mast cells and basophils. Curr. Opin. Hematol. 7:32-39.

21. Tchougounova, E., Forsberg, E., Angelborg, G., Kjellen, L., and Pejler, G. 2000 . Altered processing of fibronectin in mice lacking heparin. A role for heparin-dependent mast cell chymase in fibronectin degradation. $J$. Biol. Chem. In press.

22. Costell, M., et al. 1999. Perlecan maintains the integrity of cartilage and some basement membranes. J. Cell Biol. 147:1109-1122.

23. Arikawa-Hirasawa, E., Watanabe, H., Takami, H., Hassell, J.R., and Yamada, Y. 1999. Perlecan is essential for cartilage and cephalic development. Nat. Genet. 23:354-358.

24. Timpl, R., and Brown, J.C. 1996. Supramolecular assembly of basement membranes. Bioessays. 18:123-132.

25. Mongiat, M., et al. 2000. The protein core of the proteoglycan perlecan binds specifically to fibroblast growth factor-7. J. Biol. Chem. 275:7095-7100.

26. Mongiat, M., et al. 2001. Fibroblast growth factor-binding protein is a novel partner for perlecan protein core. J. Biol. Chem. In press.

27. Nicole, S., et al. 2000. Perlecan, the major proteoglycan of basement membranes, is altered in patients with Schwartz-Jampel syndrome (chondrodystrophic myotonia) Nat. Genet. 26:480-483.
28. Erickson, A.C., and Couchman, J.R. 2000. Still more complexity in mammalian basement membranes. J. Histochem. Cytochem. 48:1291-1306.

29. Gautam, M., et al. 1996. Defective neuromuscular synaptogenesis in agrin-deficient mutant mice. Cell. 85:525-535.

30. Iozzo, R.V., and San Antonio, J.D. 2001. Heparan sulfate proteoglycans: heavy hitters in the angiogenesis arena. J. Clin. Invest. In press.

31. Alexander, C.M., et al. 2000. Syndecan-1 is required for Wnt-1-induced mammary tumorigenesis in mice. Nat. Genet. 25:329-332.

32. Lin, X., and Perrimon, N. 1999. Dally cooperates with Drosophila Frizzled 2 to transduce Wingless signalling. Nature. 400:281-284.

33. Lander, A.D., and Selleck, S.B. 2000. The elusive functions of proteoglycans: in vivo veritas. J. Cell Biol. 148:227-232.

34. Cano-Gauci, D.F., et al. 1999. Glypican-3-deficient mice exhibit developmental overgrowth and some of the abnormalities typical of Simpson-Golabi-Behmel syndrome. J. Cell Biol. 146:255-264.

35. Pilia, G., et al. 1996. Mutations in GPC3, a glypican gene, cause the Simpson-Golabi-Behmel overgrowth syndrome. Nat. Genet. 12:241-247.

36. Filmus, J., and Selleck, S.B. 2001. Glypicans: proteoglycans with a surprise. J. Clin. Invest. In press.

37. Ishiguro, K., et al. 2000. Syndecan-4 deficiency impairs focal adhesion formation only under restricted conditions. J. Biol. Chem. 275:5249-5252.

38. Echtermeyer, F., et al. 2001. Delayed wound repair and impaired angiogenesis in mice lacking syndecan-4. J. Clin. Invest. 107:R9-R14.

39. Woods, A., and Couchman, J.R. 1998. Syndecans: synergistic activators of cell adhesion. Trends Cell Biol. 8:189-192.

40. Ishiguro, K., et al. 2000. Syndecan-4 deficiency impairs the fetal vessels in the placental labyrinth. Dev. Dyn. 219:539-544.

41. Perrimon, N., and Bernfield, M. 2000. Specificities of heparan sulphate proteoglycans in developmental processes. Nature. 404:725-728.

42. Dono, R., Texido, G., Dussel, R., Ehmke, H., and Zeller, R. 1998. Impaired cerebral cortex development and blood pressure regulation in FGF-2deficient mice. EMBO J. 17:4213-4225.

43. Shworak, N., et al. 2000. 3-OST-1 deficient mice lack an obvious procoagulant phenotype. Glycobiology. 10:20. (Abstr.)

44. Iozzo, R.V. 1998. Matrix proteoglycans: from molecular design to cellular function. Annu. Rev. Biochem. 67:609-652. 